\title{
Simulation of Gas Transport in Tight/Shale Gas Reservoirs by a Multicomponent Model Based on PEBI Grid
}

\author{
Longjun Zhang, ${ }^{1}$ Daolun Li, ${ }^{1,2}$ Lei Wang, ${ }^{3}$ and Detang Lu ${ }^{1}$ \\ ${ }^{1}$ Department of Modern Mechanics, University of Science and Technology of China, Hefei 230027, China \\ ${ }^{2}$ Hefei University of Technology, Hefei 230026, China \\ ${ }^{3}$ Institute of Nuclear Energy Safety Technology, Chinese Academy of Sciences, Hefei 230031, China
}

Correspondence should be addressed to Daolun Li; ldaol@mail.ustc.edu.cn

Received 4 August 2014; Accepted 11 September 2014

Academic Editor: Peng Xu

Copyright (C) 2015 Longjun Zhang et al. This is an open access article distributed under the Creative Commons Attribution License, which permits unrestricted use, distribution, and reproduction in any medium, provided the original work is properly cited.

\begin{abstract}
The ultra-low permeability and nanosize pores of tight/shale gas reservoir would lead to non-Darcy flow including slip flow, transition flow, and free molecular flow, which cannot be described by traditional Darcy's law. The organic content often adsorbs some gas content, while the adsorbed amount for different gas species is different. Based on these facts, we develop a new compositional model based on unstructured PEBI (perpendicular bisection) grid, which is able to characterize non-Darcy flow including slip flow, transition flow, and free molecular flow and the multicomponent adsorption in tight/shale gas reservoirs. With the proposed model, we study the effect of non-Darcy flow, length of the hydraulic fracture, and initial gas composition on gas production. The results show both non-Darcy flow and fracture length have significant influence on gas production. Ignoring nonDarcy flow would underestimate $67 \%$ cumulative gas production in lower permeable gas reservoirs. Gas production increases with fracture length. In lower permeable reservoirs, gas production increases almost linearly with the hydraulic fracture length. However, in higher permeable reservoirs, the increment of the former gradually decreases with the increase in the latter. The results also show that the presence of $\mathrm{CO}_{2}$ in the formation would lower down gas production.
\end{abstract}

\section{Introduction}

Gas production from unconventional gas reservoirs, such as tight gas/shale gas reservoir, has grown great interest in recent years. Because of the ultra-low permeability (usually under $0.1 \mathrm{mD}$ ) and small pore diameter (usually under $50 \mathrm{~nm}$ ) [1], gas flow in such tight formations reveals multiflow mechanisms that cannot be described by traditional Darcy's law, such as slip flow and Knudsen diffusion $[2,3]$.

Some modeling work has been conducted to study flow mechanisms in such reservoirs. Javadpour [2] combined convective flow and Knudsen diffusion into gas mass balance equation and found that the apparent permeability derived from the new mass balance equation can lead to one to two orders of magnitude difference from the intrinsic permeability in origin Darcy's law. Beskok and Karniadakis [4] derived a unified Hagen-Poiseuille-type equation for volumetric gas flow through a single pipe. Based on Beskok and Karniadakis [4], Florence et al. [5] proposed a formulation of apparent permeability in terms of Knudsen number. Civan [6] improved the function of the dimensionless rarefaction coefficient proposed by Beskok and Karniadakis [4] and established a mathematical model for gas flow in tight gas formation [7]. Zheng et al. [8-10] proposed a predictive model for gas slippage factor and gas diffusivity in microporous media based on fractal theory. Freeman et al. [11] incorporated the dusty-gas model into TOUGH+ family code to study gas flow behavior in tight gas/shale gas reservoirs. Freeman et al. [12] also incorporate extended Langmuir isotherm into a compositional model to represent gas desorption in shale. However, they did not consider multiflow mechanisms this time, such as slip flow and transition flow. Clarkson et al. [13] modeled transport in tight gas/shale using dynamic slippage concept which developed by Ertekin et al. [14]. Swami et al. [15] and Li et al. [16] both separately incorporated multiflow mechanisms into a numerical model to simulate gas behavior in shale. Yao et al. [17] compared gas production predicted by Civan [6], 
Javadpour [2], and Dusty-gas model and studied effect of fracture parameters on gas production in shale.

However, most models above are single component model and are based on structured grid [2, 7, 13, 1517]. Some models $[2,11,12]$ did not combine multiflow mechanisms and gas sorption together. In this paper, we first developed a compositional model which incorporates multiflow mechanisms and multicomponent adsorption based on PEBI (perpendicular bisection) grid. We also studied effect of apparent permeability, initial gas composition, and fracture length on gas production under various intrinsic permeability conditions.

\section{Mathematical Model}

2.1. PEBI Grid. PEBI grids are also known as Voronoi cells and are defined as the region in which all points are closer to the corresponding seed than any other seeds [1820]. The boundary of each Voronoi cell is normal to the line connecting the seeds on the two sides. Compared to structured Cartesian and Corner gird, the unstructured PEBI grids have the following advantages.

(1) Flexibility: it can represent the characteristic of complex boundary, pinch-out, and faults in the formation precisely.

(2) Easiness of local refinement: because of the flexibility of PEBI grid and the arbitrariness of arranging PEBI grid point, it is easier to refine grid in the local place, such as domain around wells.

(3) Less grid orientation effect: the unstructured hexagonal PEBI grid makes the grid orientation effect less significant than structured grid.

(4) Easiness of discretizing and solving equations: the local orthogonality of PEBI grid makes it easier to discretize and solve equations by using finite volume method.

The PEBI grid used in this paper is based on previous researches [21,22]. The grid points are arranged following streamline and based on well type, location, and reservoir geometry. The generated grids are denser near wells and looser far away from wells, as shown in Figure 1. This arrangement of PEBI grids can keep computation accuracy and save computation time.

2.2. Apparent Permeability. Gas flow in low-permeability tight and shale gas reservoirs occurs following various mechanisms, such as slip flow, transition flow, and free molecular flow. The matrix permeability in such reservoirs needs to be modified to enable traditional Darcy's law describe such nonDarcy flow.

Note that the non-Darcy flow in the paper includes slip flow, transition flow, and free molecular flow which is defined as below.

Chambre and Schaaf [23] have classified four flow regimes based on Knudsen number $\left(K_{n}\right)$, as shown in Table 1.
The Knudsen number $K_{n}$ expresses the mean free path of molecules as a fraction of a representative path (mean hydraulic radius, e.g.) [24]:

$$
K_{n}=\frac{\lambda_{g}}{R_{h}} .
$$

Here, $\lambda_{g}$ is the mean free path for gas and is defined by the following equation:

$$
\lambda_{g}=\frac{\mu_{g}}{P} \sqrt{\frac{\pi R T}{2 M_{g}}},
$$

where $\mu_{g}$ is the gas viscosity in Pa.s, $P$ is the absolute gas pressure in $\mathrm{Pa}, R$ is the universal gas constant $(8,314 \mathrm{~J} /(\mathrm{kmol} \cdot \mathrm{K}))$, $T$ is the absolute temperature in Kelvin, and $M_{g}$ is the molecular weight of the gas in $\mathrm{kg} / \mathrm{kmol}$.

The mean hydraulic radius of flow tubes in porous media $R_{h}$ is defined as

$$
R_{h}=2 \sqrt{2 \tau} \sqrt{\frac{k_{0}}{\phi}}
$$

where $\tau$ is the tortuosity, $\phi$ is the porosity, and $k_{0}$ is the intrinsic permeability of the reservoir in $\mathrm{m}^{2}$.

Based on the unified model for gas flow in microtubes derived by Beskok and Karniadakis [4] and Florence et al. [5] proposed a formulation of apparent permeability in terms of Knudsen number to characterize the non-Darcy flow in the porous media. Consider

$$
k=k_{0}\left(1+\alpha K_{n}\right)\left(1+\frac{4 K_{n}}{1+K_{n}}\right),
$$

where $\alpha$ is the dimensionless rarefaction coefficient and is defined by Beskok and Karniadakis as

$$
\alpha=\frac{128}{15 \pi^{2}} \tan ^{-1}\left(4 K_{n}^{0.4}\right) .
$$

Substituting (5) into (4) yields

$$
k=k_{0}\left(1+\frac{128}{15 \pi^{2}} \tan ^{-1}\left(4 K_{n}^{0.4}\right) K_{n}\right)\left(1+\frac{4 K_{n}}{1+K_{n}}\right) .
$$

This equation is valid for all flow regimes for gas flow in porous media $[4,5]$.

2.3. Multicomponent Langmuir Isotherm. To simulate and distinguish sorption capacity for different components, the extended Langmuir isotherm which is widely accepted by petroleum industry is used.

For component $i$, the sorption volume is as follows:

$$
V_{\mathrm{ads}, i}=V_{L, i} \frac{y_{i} P}{P_{L, i}\left(1+\sum_{j=1}^{n_{h}} y_{j}\left(P / P_{L, j}\right)\right)}, \quad i=1, \ldots, n_{h},
$$

where $V_{\mathrm{ads}, i}$ is the standard volume of sorbed component $i, y_{i}$ is the mole fraction of the component $i$, and $n_{h}$ is 
TABLE 1: Flow regimes classified by Chambre and Schaaf.

\begin{tabular}{lcccc}
\hline$K_{n}$ & $>10.0$ & $(0.1,10)$ & $\left(10^{-3}, 0.1\right)$ & $<10^{-3}$ \\
\hline Flow regimes & Free molecular flow & Transition flow & Slip flow & Continuum flow \\
\hline
\end{tabular}

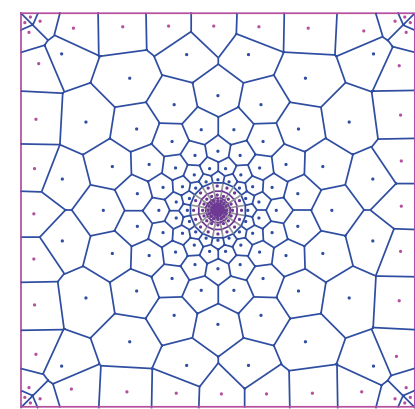

(a) One vertical well in the middle

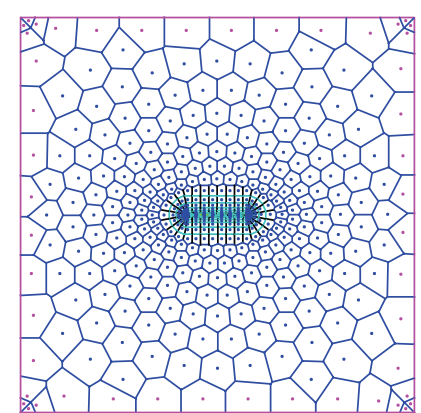

(b) One vertical well with a hydraulic fracture in the middle

Figure 1: Schematic of PEBI grid.

the total number of components. The Langmuir volume $V_{L, i}$ and Langmuir pressure $P_{L, i}$ are measured values for the pure component $i$. The total sorption is given by

$$
V_{\mathrm{ads}}=\sum_{i=1}^{n_{h}} V_{\mathrm{ads}, i}=\sum_{i=1}^{n_{h}} V_{L, i} \frac{y_{i} P}{P_{L, i}\left(1+\sum_{j=1}^{n_{h}} y_{j}\left(P / P_{L, j}\right)\right)}
$$

2.4. Mass Conservation Equations. Based on finite volume method, the governing mass balance equation for the component $i$ considering gas sorption is given by

$$
\begin{aligned}
& \frac{\partial}{\partial t}\left(V \phi \rho_{g} y_{i}+V \rho_{s} V_{\mathrm{ads}, i} \rho_{g, \mathrm{std}}\right) \\
&=\sum_{l}\left(T_{r} \frac{1}{\mu_{g}} \rho_{g} y_{i} \Delta \Phi_{g}\right)_{l}-\rho_{g, \text { std }} y_{i} q_{g, \text { std }}, \\
& i=1, \ldots, n_{h},
\end{aligned}
$$

where $V$ is the gas volume in $\mathrm{m}^{3}, \rho_{s}$ is the rock density in $\mathrm{kg} / \mathrm{m}^{3}, \rho_{g \text {,std }}$ is the gas density under standard conditions $\left(1 \mathrm{~atm}\right.$ and $\left.15^{\circ} \mathrm{C}\right)$ in $\mathrm{mol} / \mathrm{m}^{3}, \rho_{g}$ is the gas density under formation condition in $\mathrm{mol} / \mathrm{m}^{3}$, and $q_{g \text {,std }}$ is the gas production rate under standard condition in $\mathrm{m}^{3} / \mathrm{s}$. Values of $\rho_{g}$ and $\rho_{g \text {,std }}$ were calculated using the Peng-Robinson equation of state (PR EOS) [25]. The symbol $l$ represents connection between adjacent grids, and $\Delta \Phi_{g}$ is the difference in gas potentials between adjacent grids $l 1$ and $l 2$ and is given by $\Delta \Phi_{g}=\Phi_{l 1}-\Phi_{l 2}=p_{l 1}-p_{l 2}-\left(\rho_{l 1} Z_{l 1}-\rho_{l 2} Z_{l 2}\right) g$, where $Z$ is the depth of the formation. The viscosity $\mu_{g}$ was calculated using the Lohrenz-Bray-Clark (LBC) correlation [26]. The transmissibility parameter $T_{r}=k A / \Delta L$, where $k$ is the apparent permeability represented by (6), $A$ is the cross-sectional area between the adjacent grids, and $\Delta L$ is the distance between the adjacent grids.

The left term of (9) is the mass accumulation term and includes the mass of both free gas and sorbed gas. The first right term denotes the advection term. The second right term denotes source or sink in the well, which stands for the rate of gas mass produced from or injected into a well.

For vertical well, the well production rate $q_{g \text {,std }}$ is expressed by Peaceman model:

$$
q_{g, \text { std }}=\frac{\theta k h}{\mu_{g} \ln \left(r_{e} / r_{w}\right)}\left(p_{j}-p_{w f}\right),
$$

where $h$ is the effective height of well perforation in $\mathrm{m}, r_{w}$ is wellbore radius in $\mathrm{m}, p_{j}$ is well grid pressure in $\mathrm{Pa}, p_{w f}$ is bottom-hole flowing pressure in $\mathrm{Pa}$, and $r_{e}$ is the equivalent radius in the well grid in $\mathrm{m}$. For structured Cartesian grid, $r_{e}$ can be expressed as

$$
r_{e}=0.28 \frac{\left[\left(k_{y} / k_{x}\right)^{0.5} \Delta x^{2}+\left(k_{x} / k_{y}\right)^{0.5} \Delta y^{2}\right]^{0.5}}{\left(k_{y} / k_{x}\right)^{0.25}+\left(k_{x} / k_{y}\right)^{0.25}} .
$$

For unstructured PEBI grid, we derive $r_{e}$ as follows.

Assume pressure at equivalent radius $r_{e}$ as $p_{e}$ which is equivalent to well grid pressure $p_{j}$. Arranging (10) yields

$$
\left(p_{e}-p_{w f}\right)=\frac{\mu_{g} \ln \left(r_{e} / r_{w}\right) q_{g, \text { std }}}{\theta k h} .
$$

The flux from adjacent grids to well grid $Q$ is the same as $q_{g \text {,std }}$. $Q$ can be expressed as

$$
Q=q_{g, \text { std }}=\sum_{l} \frac{T_{r}}{\mu_{g}}\left(p_{l}-p_{j}\right)=\sum_{l} \frac{T_{r}}{\mu_{g}}\left(p_{l}-p_{e}\right) .
$$

Arranging (10) and (12) and substituting into (13) yield

$$
\ln r_{e}=\frac{\sum_{l} T_{r} \ln \Delta L-\theta k h}{\sum_{l} T_{r}} .
$$

Consequently, $r_{e}$ can be expressed as follows:

$$
r_{e}=\exp \left(\frac{\sum_{l} T_{r} \ln \Delta L-\theta k h}{\sum_{l} T_{r}}\right) .
$$


TABLE 2: Properties and parameters of a tight gas reservoir in Xinjiang, China.

\begin{tabular}{lcc}
\hline Name & Value & Units \\
\hline Reservoir dimensions & $3500 \times 2000 \times 18$ & Meter \\
Fracture half-length & 230 & Meter \\
Permeability & 0.056 & $\mathrm{mD}$ \\
Porosity & 0.0376 & $\mathrm{~N} / \mathrm{A}$ \\
Reservoir temperature & 355.0 & $\mathrm{Kelvin}$ \\
Initial pressure & 31.5 & $\mathrm{MPa}$ \\
Bulk density & 2500.0 & $\mathrm{~kg} / \mathrm{m}^{3}$ \\
Wellbore diameter & 0.084 & $\mathrm{~meter}$ \\
Langmuir volume $\left(V_{L}\right)$ & 0.0012 & $\mathrm{~m}^{3} / \mathrm{kg}$ \\
Langmuir pressure $\left(P_{L}\right)$ & 7.3 & $\mathrm{MPa}$ \\
\hline
\end{tabular}

For vertical well with a hydraulic fracture, we use the infinite conductivity model [27] to represent the conductivity of the fracture in the reservoir. Considering the wellbore storage, the well production rate $q_{g \text {,std }}$ can be expressed as

$$
q_{g, \text { std }}=\frac{\sum_{l}\left(\left(T_{r} / \mu_{g}\right) \rho_{g} \Delta \Phi_{g}\right)_{l}}{\rho_{g, \text { std }}}-\frac{C}{\Delta t}\left(p_{w f}^{t_{n+1}}-p_{w f}^{t_{n}}\right),
$$

where $C$ is the wellbore storage in $\mathrm{m}^{3} / \mathrm{MPa}$ and $t^{n+1}$ and $t^{n}$ are $(n+1)$ th and $n$th time steps.

The unknown variables include pressure $p$, bottom-hole flowing pressure $p_{w f}$, and the mole fraction $y_{i}$ in mass conservation equations (9). The nonlinear equations are solved using Newton iteration method and the matrix solver GMRES (generalized minimal residual method) [28].

\section{Model Validation}

The model was validated by reproducing the pressure buildup data from a hydraulic fractured vertical well in a tight gas reservoir in Xinjiang, China. The pilot test operation comprised two stages: gas production at the rate of $8.36 \times$ $10^{4} \mathrm{~m}^{3}$ /day for 30 days, followed by a shut-in period of 25 days.

The model was set up using parameters and properties listed in Table 2. The initial reservoir gas contains $94.7 \%$ $\mathrm{CH}_{4}, 1.2 \% \mathrm{CO}_{2}, 2.7 \% \mathrm{C}_{2} \mathrm{H}_{6}$, and $1.4 \% \mathrm{C}_{3} \mathrm{H}_{8}$. The average gas sorption parameters $\left(V_{L}\right.$ and $\left.P_{L}\right)$ in Table 2 were provided without distinguishing different type of gas components. Figure 2 shows the predicted pressure dropdown during the production stage and the pressure build-up during the shut-in period. The modeling output was compared to the monitored high frequency/high-resolution BHP data during the shut-in operation [29]. The pressure data during the production was not available. The simulation output shows reasonable reproduction of the pressure build-up data.

\section{Results and Discussion}

The developed model was used to understand the effect of non-Darcy flow, length of the hydraulic fracture, and initial gas composition on gas production.

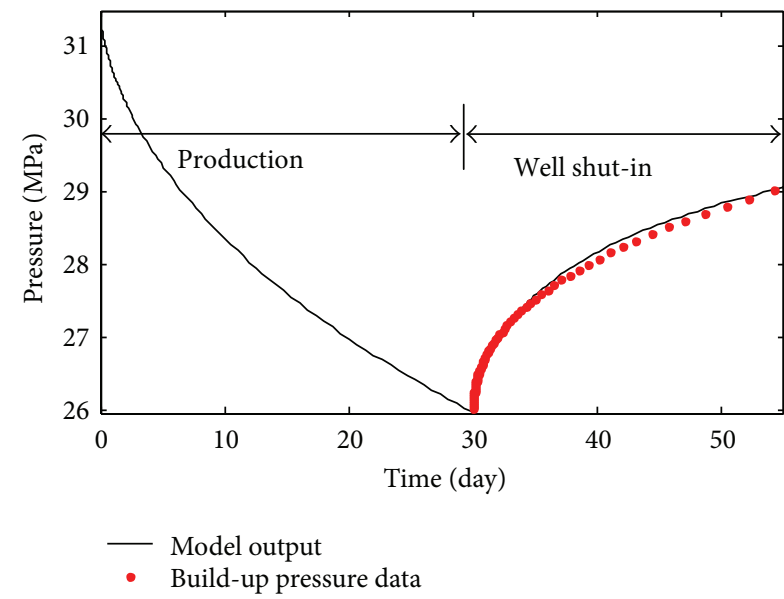

FIGURE 2: The bottom-hole pressure history of a production well in a tight gas reservoir in Xinjiang, China. The production rate from day 0 to 30 was $8.36 \times 10^{4} \mathrm{~m}^{3} /$ day, where pressure data was not available. The predicted pressure build-up (black line) during the shut-in period compares well with data (red symbol).

Specific reservoir parameters and simulation conditions are listed in Table 3, unless noted otherwise. The sorption parameters for gas species are listed in Table 4 . The hydraulically fractured vertical well is located in the middle of simulated gas reservoir of which the boundary is sealed.

4.1. The Effect of Non-Darcy Flow on Gas Production. Here, the gas production was calculated with and without considering non-Darcy flow under three different intrinsic permeability conditions which are $1.01 \times 10^{-2} \mathrm{mD}\left(10^{-17} \mathrm{~m}^{2}\right)$, $1.01 \times 10^{-4} \mathrm{mD}\left(10^{-19} \mathrm{~m}^{2}\right)$, and $1.01 \times 10^{-6} \mathrm{mD}\left(10^{-21} \mathrm{~m}^{2}\right)$, respectively.

The non-Darcy flow is incorporated into apparent permeability which is represented by (6). The non-Darcy flow in tight formations includes slip flow, transition flow, and free molecular flow which will enhance the gas flow ability, especially under lower pressure condition. Thus, as gas produces, the reservoir pressure continues to decrease and the influence of non-Darcy flow on gas production will become more significant.

In the higher permeable reservoir $\left(k_{0}=1.01 \times 10^{-2} \mathrm{mD}\right)$, the influence of non-Darcy flow on gas production is very limited, as depicted by Figure 3(a). The flow regime of gas mainly stays in slip flow regime. After $4.38 \times 104$ days $(120$ years) production, the difference of predicted produced gas volume with and without considering non-Darcy flow is only about 2\%, as shown in Figure 4.

While in lower permeable gas reservoirs $\left(k_{0}=1.01 \times\right.$ $10^{-4} \mathrm{mD}, k_{0}=1.01 \times 10^{-6} \mathrm{mD}$ ) which are more common for tight/shale gas reservoirs, the non-Darcy flow (mainly under transition flow regime) reveals more significant influence on produced gas volume, as depicted in Figures 3(b) and 3 (c). The predicted produced gas volume without considering non-Darcy flow is $24 \%$ and $67 \%$ smaller than that with considering non-Darcy flow for reservoir $k_{0}=1.01 \times 10^{-4} \mathrm{mD}$ and reservoir $k_{0}=1.01 \times 10^{-6} \mathrm{mD}$, respectively. 
TABLE 3: Input parameters for simulated hydraulically fractured vertical well and the gas reservoir.

\begin{tabular}{lcc}
\hline Input parameter & Values & Unit \\
\hline Dimension & $2000 \times 1000 \times 25$ & $\mathrm{~m}$ \\
Fracture half-length & 200 & $\mathrm{~m}$ \\
Wellbore storage & 1 & $\mathrm{~m}^{3} / \mathrm{MPa}$ \\
Flowing bottom-hole pressure & 10 & $\mathrm{MPa}$ \\
Porosity & 0.05 & \\
Temperature & 60 & ${ }^{\circ} \mathrm{C}$ \\
Initial reservoir pressure & 35 & $\mathrm{MPa}$ \\
Initial gas composition & $2 \% \mathrm{CO}_{2}, 90 \% \mathrm{CH}_{4}, 8 \% \mathrm{C}_{2} \mathrm{H}_{6}$ & $\mathrm{Kg} / \mathrm{m}^{3}$ \\
Bulk density & 2500 & \\
\hline
\end{tabular}

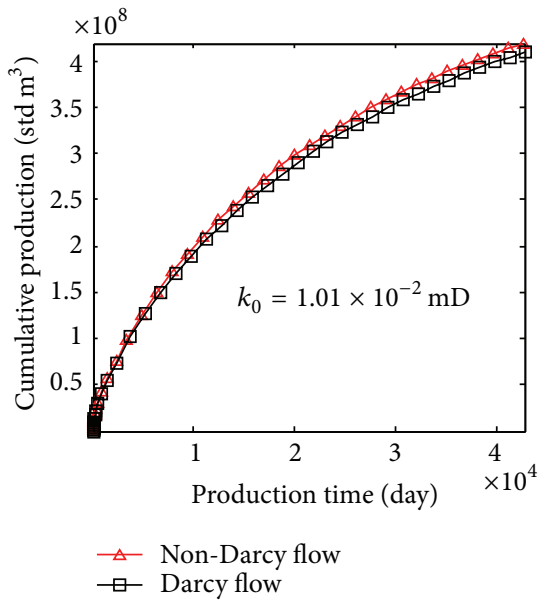

(a)

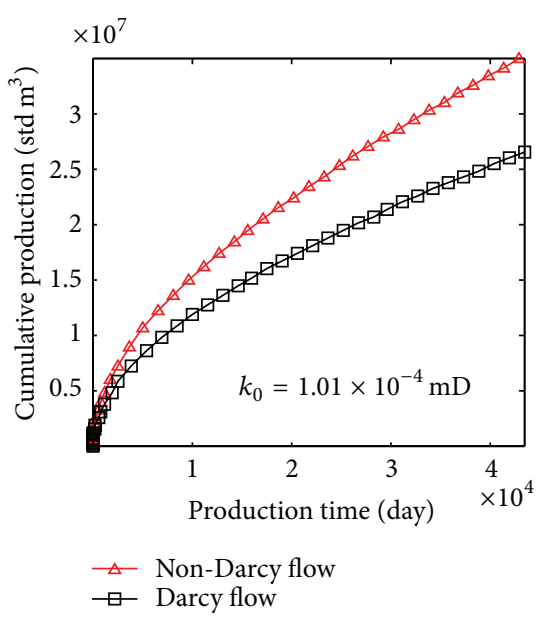

(b)

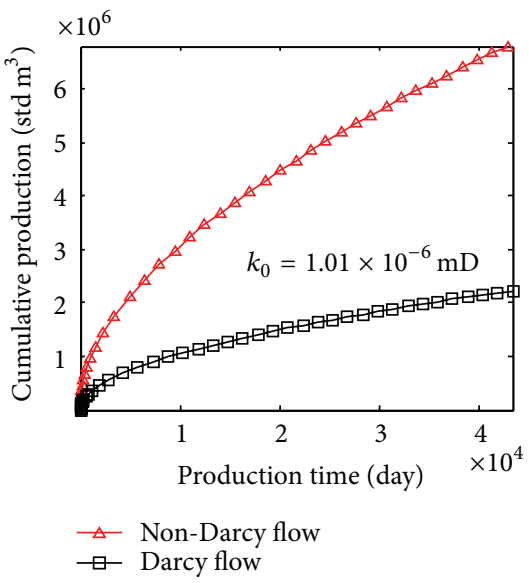

(c)

FIGURE 3: The gas production predicted with and without considering non-Darcy flow under three different intrinsic permeability conditions.

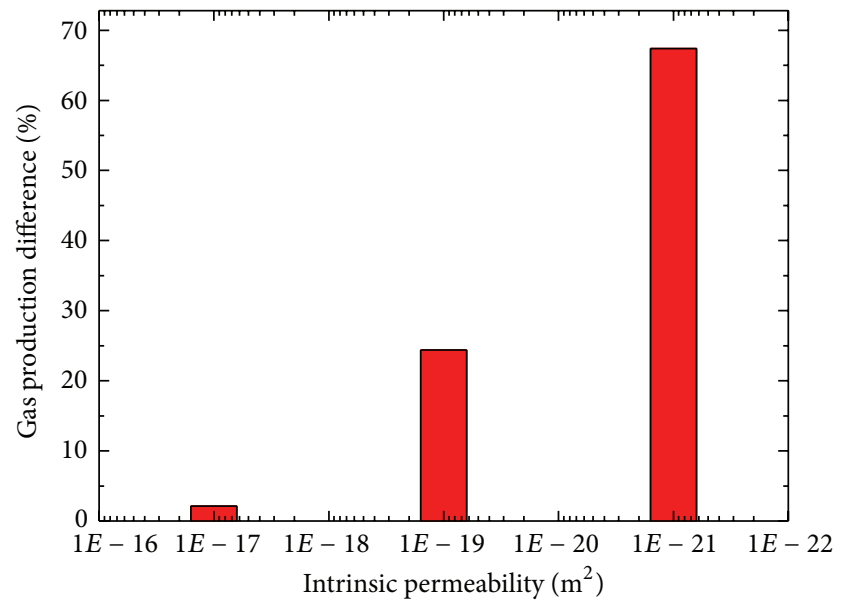

FIGURE 4: Gas production difference predicted with considering non-Darcy flow and without considering it after 120 years of production.

Lower permeability often indicates smaller pore diameter which means that more chance of non-Darcy flow would happen during gas flow. From Figures 3(a) to 3(c), as the permeability goes smaller, the gas flow regimes mainly stay
TABLE 4: Langmuir constants for gas species in gas reservoir.

\begin{tabular}{lccc}
\hline & $\mathrm{CO}_{2}$ & $\mathrm{CH}_{4}$ & $\mathrm{C}_{2} \mathrm{H}_{6}$ \\
\hline$V_{L}\left(\mathrm{~m}^{3} / \mathrm{t}\right)$ & 4.1 & 1.6 & 2.6 \\
$P_{L}(\mathrm{MPa})$ & 5.76 & 10.77 & 5.59 \\
\hline
\end{tabular}

on slip flow, early transition flow, and late transition flow, respectively.

Based on the results above, non-Darcy flow is critical for accurate predicting gas flow behavior in low permeable reservoirs, such as tight gas or shale gas reservoirs. Ignoring it would significantly underestimate gas production capacity.

4.2. The Effect of Length of Hydraulic Fracture on Gas Production. Here, we aim to understand the role of hydraulic fracture length in determining gas production. Gas production rate and cumulative gas production were predicted when the half-length of hydraulic fracture equals $0,50,100,200$, and 400 meters in the reservoir of which intrinsic permeability equals $1.01 \times 10^{-2} \mathrm{mD}, 1.01 \times 10^{-4} \mathrm{mD}$, and $1.01 \times 10^{-6} \mathrm{mD}$.

Take the medium permeable reservoir $\left(k_{0}=1.01 \times\right.$ $10^{-4} \mathrm{mD}$ ), for example. The gas cumulative production and production rate are depicted in Figure 5. 


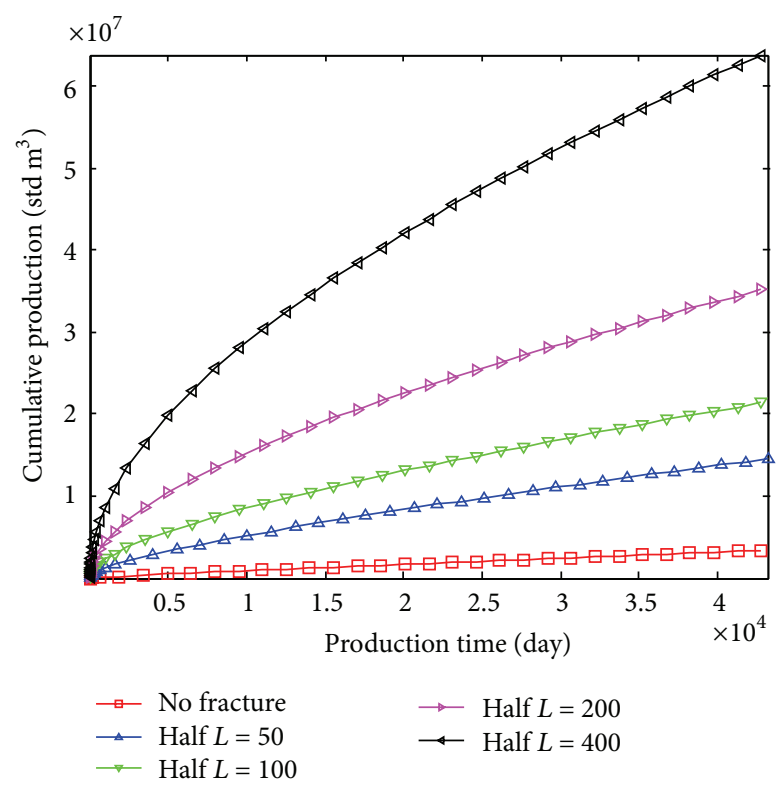

(a) Cumulative production

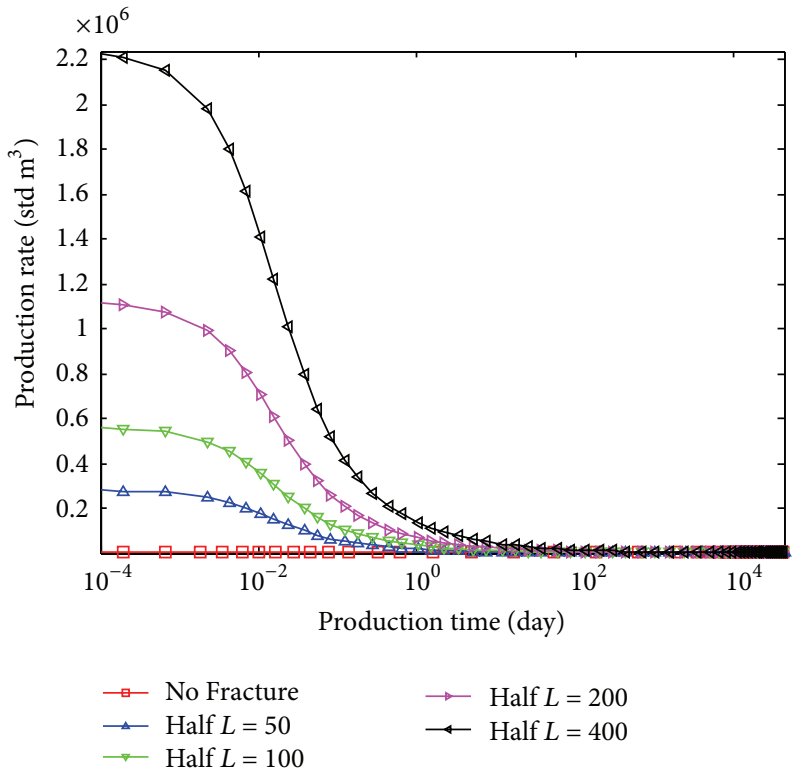

(b) Production rate

FIGURE 5: Effect of fracture half-length on predicted gas cumulative production and production rate.

The results show that gas production rate and cumulative production increase with half-length of hydraulic fracture. The vertical well with no fracture (represented by red line) indicates very limited production potential. The averaged production rate is only $79 \mathrm{~m}^{3} /$ day and the cumulative production after 120 years is only 3.4 million cubic meters, as shown in Figures 5(a) and 5(b), while for the vertical well with hydraulic fracture of which the half-length is only 50 meters, the cumulative production increases to 15 million cubic meters, about five times as that of the well with no fracture. As the half-length of fracture increases to $100 \mathrm{~m}$, $200 \mathrm{~m}$, and $400 \mathrm{~m}$, the cumulative production increases to 22,36 , and 65 million cubic meters, respectively. With the increase of half-length of fracture, the extent of cumulative production increased is remarkable, indicating the significant importance of length of hydraulic fracture on gas production.

Gas production rate in such low permeable reservoir decreases very quickly, especially in the early production time, as depicted by Figure 5(b). For the well with a fracture half-length of 400 meters, in the first 2 days of production, the rate stays above $100,000 \mathrm{~m}^{3} /$ day, while after 200 days of production, the rate decreases rapidly to $10,000 \mathrm{~m}^{3} /$ day. In the late production time, the rate decreases much slower. After 100 years of production, the rate remains fairly at $1,000 \mathrm{~m}^{3} /$ day.

For all simulated reservoirs, the increase of half-length of hydraulic fracture shows a positive influence on gas production, as depicted in Figure 6. In the higher permeable reservoir $\left(k_{0}=1.01 \times 10^{-2} \mathrm{mD}\right)$, the cumulative production shows nonlinear relationship with fracture half-length. With increase of fracture half-length, the increment of cumulative production decreases gradually and may finally become negligible. In the medium permeable reservoir $\left(k_{0}=1.01 \times\right.$ $\left.10^{-4} \mathrm{mD}\right)$, the cumulative production shows almost linear increment relationship with fracture half-length. And in the lower permeable reservoir $\left(k_{0}=1.01 \times 10^{-6} \mathrm{mD}\right)$, the relationship between the two can be identified as linear.

Thus, we conclude that the lower the permeability is, the more significant the increasing fracture length influences gas production. But for some relatively higher permeable reservoirs, an appropriate length of fracture should be found considering the balance between economical cost of fracturing a well and gas production.

\subsection{The Effect of Initial Gas Composition on Gas Production.} Here, gas cumulative productions are calculated when initial $\mathrm{CO}_{2}$ composition equals to $0 \%, 10 \%, 20 \%$, and $30 \%$, respectively. The half-length of hydraulic fracture is confined to 200 meters. The intrinsic permeability is confined to $1.01 \times$ $10^{-4} \mathrm{mD}$. The initial gas species in the reservoir is assumed to be $\mathrm{CO}_{2}$ and $\mathrm{CH}_{4}$. All other parameters are the same as in Tables 3 and 4. The results are shown in Figure 7.

The results show initial gas composition has some influence on cumulative production. Higher percentage of $\mathrm{CO}_{2}$ in the initial gas leads to lower gas production which can be seen clearly on the enlarged figure. This is mainly because the presence of $\mathrm{CO}_{2}$ increases gas viscosity, which would slow down flow speed in the formation and eventually reduce gas production.

The decrement of cumulative gas production slows down with the increase of $\mathrm{CO}_{2}$ percentage in initial gas content, as depicted in Figure 8(a), while that of cumulative methane production does not, as depicted in Figure 8(b). The cumulative methane production shows a linear relationship with the percentage of $\mathrm{CO}_{2}$ in initial gas content. When initial $\mathrm{CO}_{2}$ percentage equals $0 \%$, the produced cumulative gas volume is $4.4 \%$ ( 1.5 million $\mathrm{m}^{3}$ ) higher than that of the case when initial $\mathrm{CO}_{2}$ percentage equals $30 \%$, while for produced cumulative 


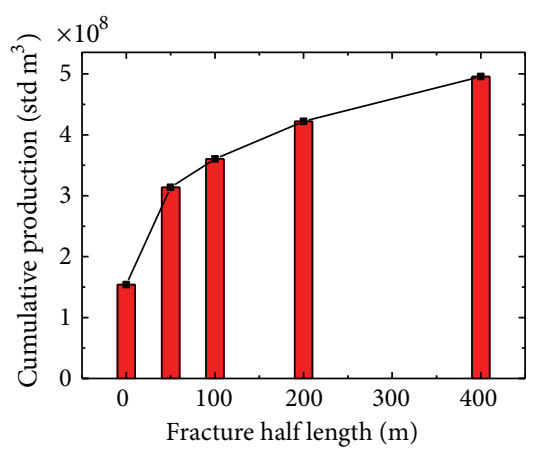

(a) $k_{0}=1.01 \times 10^{-2} \mathrm{mD}$

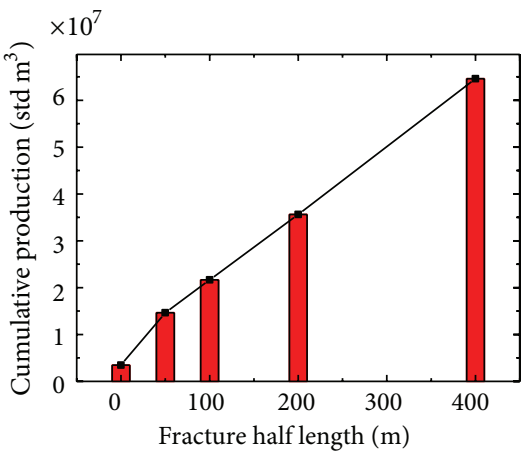

(b) $k_{0}=1.01 \times 10^{-4} \mathrm{mD}$

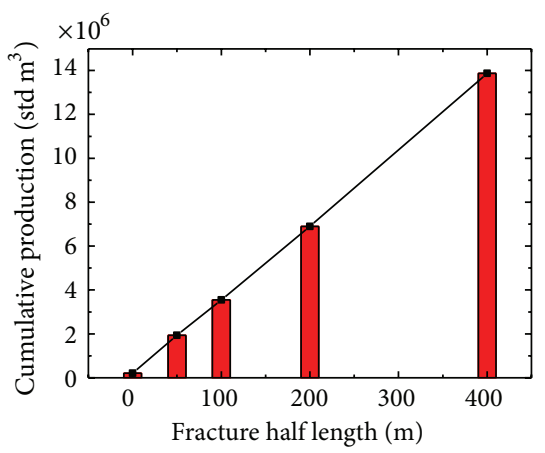

(c) $k_{0}=1.01 \times 10^{-6} \mathrm{mD}$

FIGURE 6: Effect of fracture half-length on cumulative production after 120 years of production in three different permeable gas reservoirs.

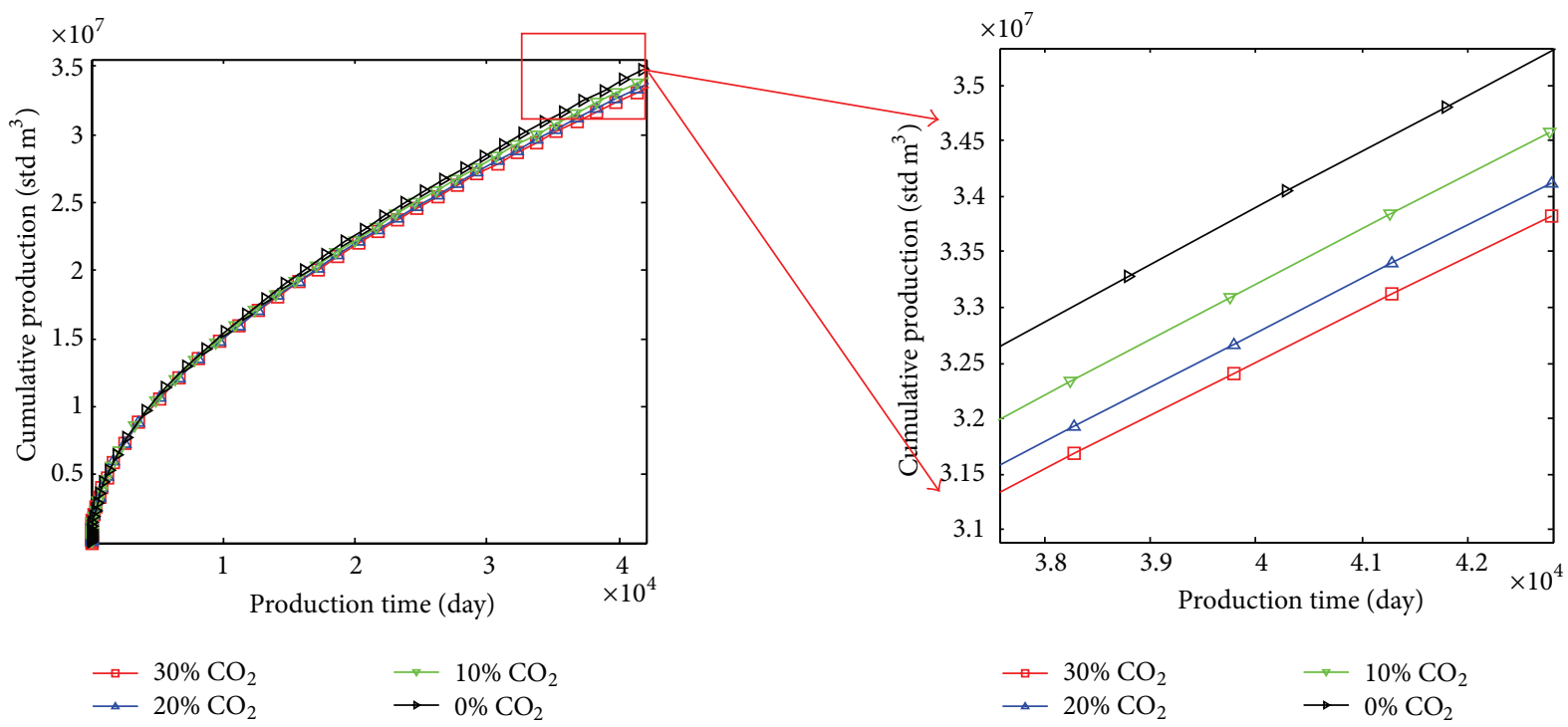

FIGURE 7: Effect of initial gas composition on cumulative production.

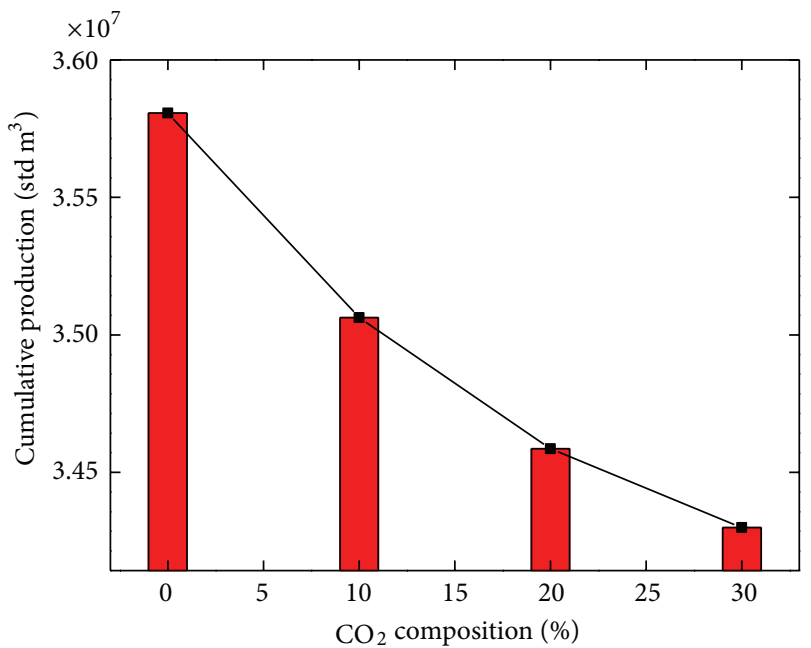

(a)

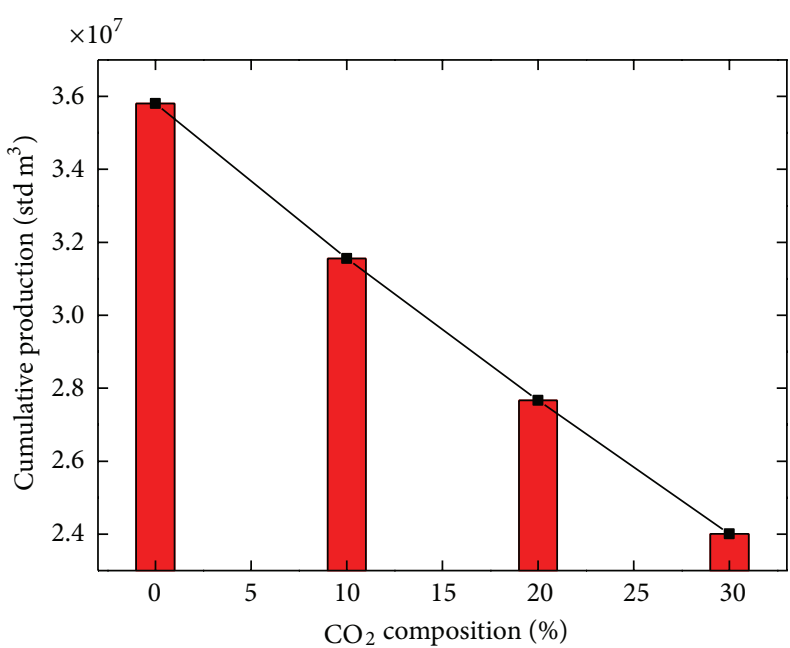

(b)

FigURE 8: Effect of initial gas composition on produced (a) cumulative mixed gas volume and (b) methane volume after 120 years of production. 
methane volume, the former is $49.8 \%$ (11.9 million $\mathrm{m}^{3}$ ) higher than the latter.

\section{Conclusions}

In this paper, we proposed a compositional model for tight/shale gas reservoirs based on unstructured PEBI grid. The non-Darcy flow, including slip flow, transition flow, and free molecular flow, is considered in terms of apparent permeability in the model. Multicomponent adsorption is also considered in terms of extended Langmuir isotherm.

With the proposed model, we studied the effect of nonDarcy flow, length of the hydraulic fracture, and initial gas composition on gas production. The results showed the following: (1) the non-Darcy flow shows significant influence on gas production, especially in low permeable reservoirs. Ignoring this effect would lead to $67 \%$ underestimation on produced cumulative gas volume according to the simulated case. (2) Gas production increases with half-length of hydraulic fracture. However, in higher permeable reservoirs, the increment of gas production decreases with increase in fracture length, while in lower permeable reservoirs, gas production almost increases linearly with fracture length. Gas production would impossibly reach to economic rate without fracturing the well. (3) Higher initial $\mathrm{CO}_{2}$ percentage would lead to lower gas production, for its ability to increase gas viscosity in the formation.

With considering non-Darcy flow and multicomponent gas adsorption in tight/shale gas reservoirs, the proposed compositional model can be a powerful tool to predict gas behavior in such unconventional reservoirs and be used to offer valuable insights into reservoir engineers to make better exploitation schemes.

\section{Conflict of Interests}

The authors declare that there is no conflict of interests regarding the publication of this paper.

\section{Acknowledgments}

The authors are grateful for funding from Major State Basic Research Development Program of China (973 Program) (no. 2011CB707305) and National Key Science and Technology Project (2011ZX05009-006).

\section{References}

[1] P. H. Nelson, "Pore-throat sizes in sandstones, tight sandstones, and shales," AAPG Bulletin, vol. 93, no. 3, pp. 329-340, 2009.

[2] F. Javadpour, "Nanopores and apparent permeability of gas flow in mudrocks (shales and siltstone)," Journal of Canadian Petroleum Technology, vol. 48, no. 8, pp. 16-21, 2009.

[3] F. Javadpour, D. Fisher, and M. Unsworth, "Nanoscale gas flow in shale gas sediments," Journal of Canadian Petroleum Technology, vol. 46, no. 10, pp. 55-61, 2007.

[4] A. Beskok and G. E. Karniadakis, "A model for flows in channels, pipes, and ducts at micro and nano scales," Microscale Thermophysical Engineering, vol. 3, no. 1, pp. 43-77, 1999.
[5] F. Florence, J. Rushing, K. E. Newsham, and T. A. Blasingame, "Improved permeability prediction relations for low permeability sands," in Proceedings of the Rocky Mountain Oil \& Gas Technology Symposium, 2007.

[6] F. Civan, "Effective correlation of apparent gas permeability in tight porous media," Transport in Porous Media, vol. 82, no. 2, pp. 375-384, 2010.

[7] F. Civan, C. S. Rai, and C. H. Sondergeld, "Shale-gas permeability and diffusivity inferred by improved formulation of relevant retention and transport mechanisms," Transport in Porous Media, vol. 86, no. 3, pp. 925-944, 2011.

[8] Q. Zheng and B. Yu, "A fractal permeability model for gas flow through dual-porosity media," Journal of Applied Physics, vol. 111, no. 2, Article ID 024316, 2012.

[9] Q. Zheng, B. Yu, S. Wang, and L. Luo, "A diffusivity model for gas diffusion through fractal porous media," Chemical Engineering Science, vol. 68, no. 1, pp. 650-655, 2012.

[10] Q. Zheng, B. Yu, Y. Duan, and Q. Fang, "A fractal model for gas slippage factor in porous media in the slip flow regime," Chemical Engineering Science, vol. 87, pp. 209-215, 2013.

[11] C. M. Freeman, G. J. Moridis, and T. A. Blasingame, " A numerical study of microscale flow behavior in tight gas and shale gas reservoir systems," Transport in Porous Media, vol. 90, no. 1, pp. 253-268, 2011.

[12] C. M. Freeman, G. J. Moridis, and T. A. Blasingame, "Modeling and performance interpretation of flowing gas composition changes in shale gas wells with complex fractures," in Proceedings of the International Petroleum Technology Conference: Challenging Technology and Economic Limits to Meet the Global Energy Demand (IPTC '13), pp. 4868-4883, Beijing, China, March 2013.

[13] C. R. Clarkson, M. Nobakht, D. Kaviani, and T. Ertekin, "Production analysis of tight-gas and shale-gas reservoirs using the dynamic-slippage concept," SPE Journal, vol. 17, no. 1, pp. 230-242, 2012.

[14] T. Ertekin, G. R. King, and F. C. Schwerer, "Dynamic gas slippage: a unique dual-mechanism approach to the flow of gas in tight formations," SPE Formation Evaluation, vol. 1, no. 1, pp. 43-52, 1986.

[15] V. Swami, F. Javadpour, and A. Settari, "A numerical model for multi-mechanism flow in shale gas reservoirs with application to laboratory scale testing," in Proceedings of the 75th EAGE Conference \& Exhibition Incorporating SPE EUROPEC, 2013.

[16] J. Li, C. Wang, D. Ding, Y. S. Wu, and Y. Di, "A generalized framework model for simulation of gas production in unconventional gas reservoirs," in Proceedings of the SPE Reservoir Simulation Symposium, The Woodlands, Tex, USA, February 2013.

[17] J. Yao, H. Sun, D.-Y. Fan, C.-C. Wang, and Z.-X. Sun, "Numerical simulation of gas transport mechanisms in tight shale gas reservoirs," Petroleum Science, vol. 10, no. 4, pp. 528-537, 2013.

[18] Z. E. Helnemann, C. W. Brand, M. Munka, and Y. M. Chen, "Modeling reservoir geometry with irregular grids," SPE Reservoir Engineering, vol. 6, no. 2, pp. 225-232, 1991.

[19] Z. E. Heinemann and C. W. Brand, "Gridding techniques in reservoir simulation," in Proceedings of the 1st International Forum on Reservoir Simulation, Alpbach, Austria, 1988.

[20] C. L. Palagi and K. Aziz, "Use of Voronoi grid in reservoir simulation," SPE Advanced Technology Series, vol. 2, no. 2, pp. 69-77, 1994. 
[21] L. Zhang, D. Li, W. Zha et al., "Generation and application of adaptive PEBI grid for numerical well testing(NWT)," in Proceedings of the International Conference on Mechatronic Sciences, Electric Engineering and Computer (MEC '13), pp. 3002-3006, Shenyang, China, 2013.

[22] W. Zha, Numerical Reservoir Calculation on PEBI Grid and Implementation, University of Science and Technology of China, 2009.

[23] P. A. Chambre and S. A. Schaaf, Flow of Rarefied Gases, 1961.

[24] L. B. Loeb, The Kinetic Theory of Gases, Courier Dover, 2004.

[25] S. M. Walas, Phase Equilibria in Chemical Engineering, vol. 4, Butterworth, Boston, Mass, USA, 1985.

[26] J. Lohrenz, B. Bray, and C. Clark, "Calculating viscosities of reservoir fluids from their compositions," Journal of Petroleum Technology, vol. 16, no. 10, pp. 1171-1176, 1964.

[27] B. Horsfield and H. M. Schulz, "Shale gas exploration and exploitation," Marine and Petroleum Geology, vol. 31, no. 1, pp. $1-2,2012$.

[28] Y. Saad and M. H. Schultz, "GMRES: a generalized minimal residual algorithm for solving nonsymmetric linear systems," SIAM Journal on Scientific and Statistical Computing, vol. 7, no. 3, pp. 856-869, 1986.

[29] W. Ding, C. Li, C. Li et al., "Fracture development in shale and its relationship to gas accumulation," Geoscience Frontiers, vol. 3, no. 1, pp. 97-105, 2012. 

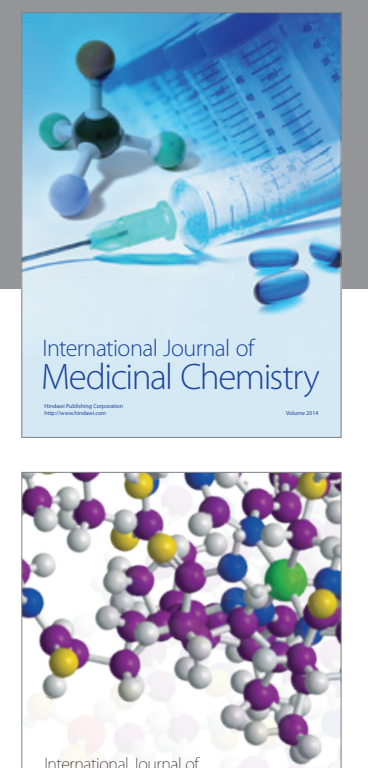

\section{Carbohydrate} Chemistry

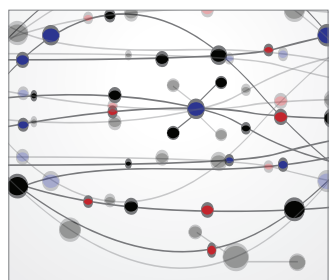

The Scientific World Journal
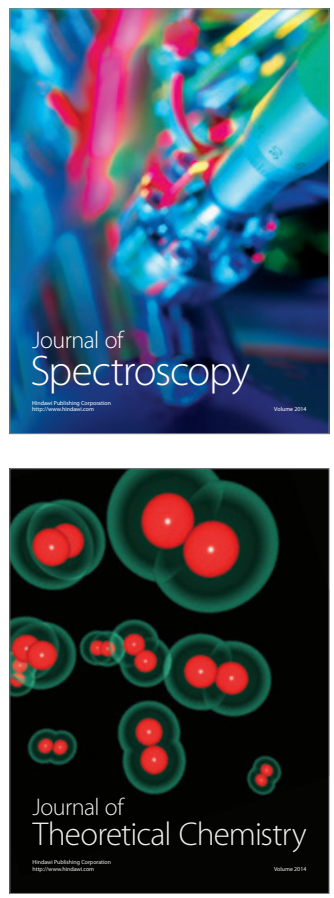
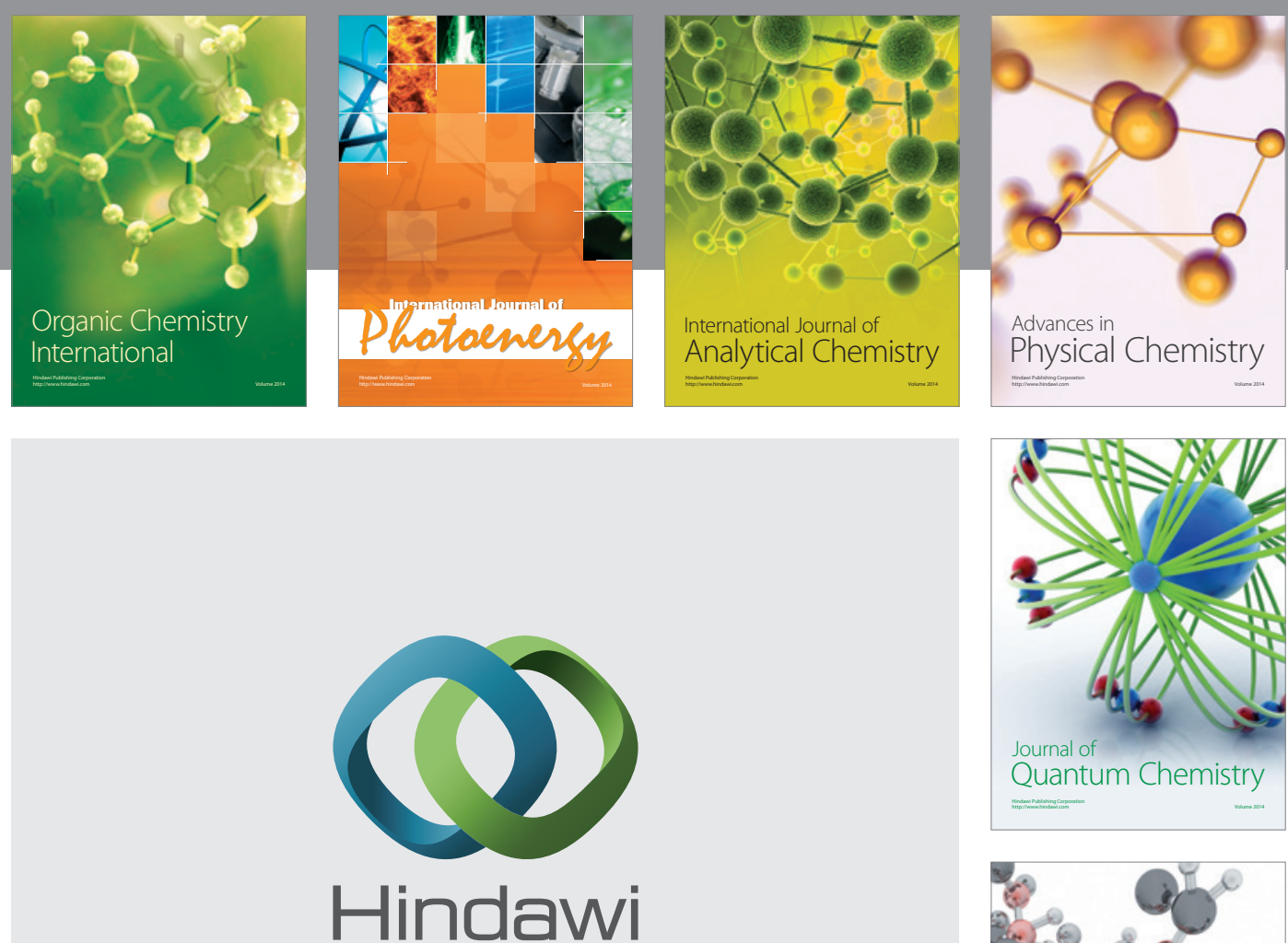

Submit your manuscripts at

http://www.hindawi.com

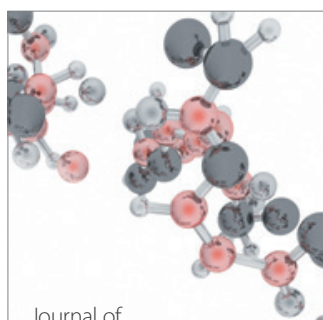

Analytical Methods

in Chemistry

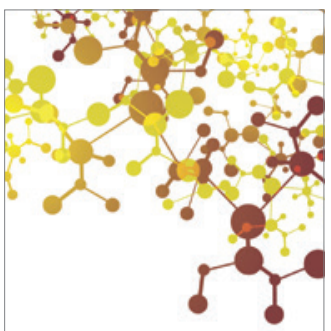

Journal of

Applied Chemistry

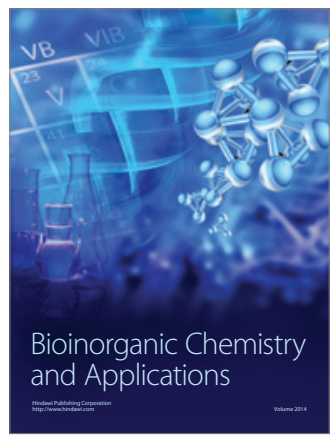

Inorganic Chemistry
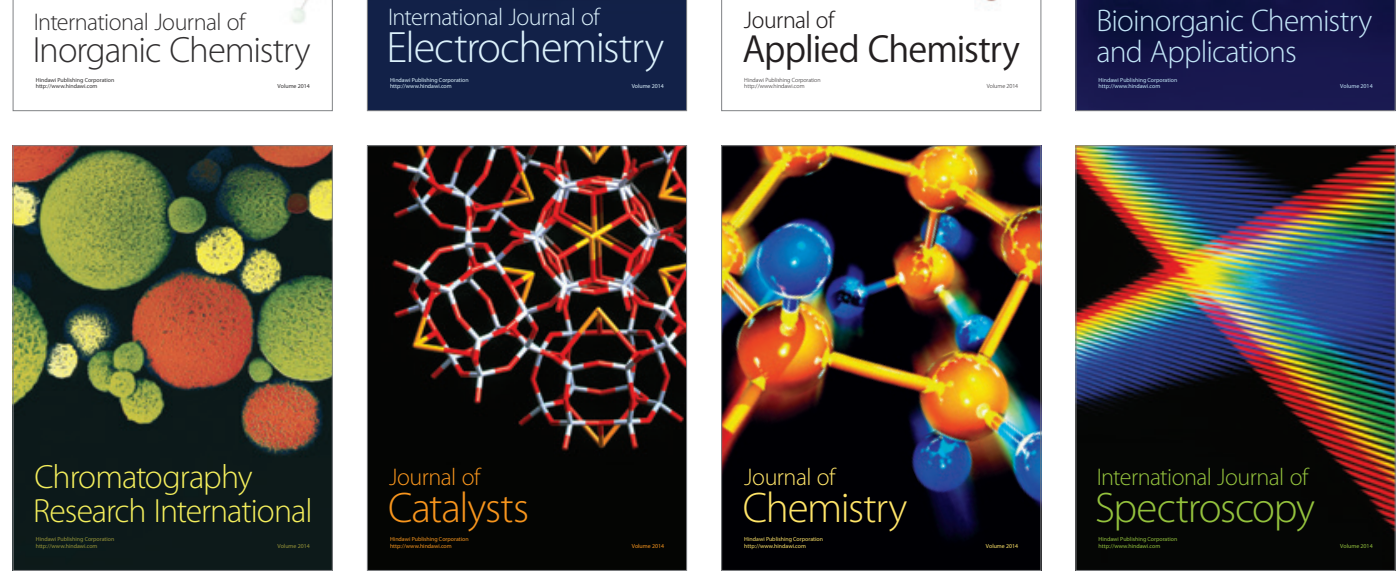